\title{
Carcinoma In Situ Involving Sclerosing Adenosis on Core Biopsy: Diagnostic Pearls to Aid the Practicing Clinician and Avoid Overtreatment
}

\author{
Dana Richards $\cdot$ Alberto A. Ayala $\cdot$ Yun Wu Lavinia P. Middleton
}

Received: September 13, 2019 / Published online: January 9, 2020

(C) The Author(s) 2020

\begin{abstract}
Introduction: Involvement of pre-existing benign lesions by ductal carcinoma in situ (DCIS) or lobular neoplasia (LN) can present difficult diagnostic challenges, and can easily cause misdiagnosis of invasive carcinoma and over-management of localized disease. Our objective was to gather the largest case series of DCIS and LN involving sclerosing adenosis (SA), and to report the characteristic features of these lesions, in order to provide histologic criteria for the diagnostician.

Methods: Our database was searched for core biopsy material diagnosed as carcinoma in situ involving adenosis. Glass slides and pathology reports were reviewed. The cases were studied
\end{abstract}

Enhanced Digital Features To view enhanced digital features for this article go to https://doi.org/10.6084/ m9.figshare.11395356.

D. Richards

Department of Pathology and Laboratory Medicine, University of Kentucky Chandler Medical Center, Lexington, KY, USA

\section{A. A. Ayala}

Department of Pathology and Genomic Medicine, Houston Methodist Hospital, Houston, TX, USA

\section{Y. Wu $\cdot$ L. P. Middleton $(\bowtie)$}

Department of Pathology, The University of Texas MD Anderson Cancer Center, Houston, TX, USA e-mail: lpmiddleton@mdanderson.org for salient features, and clinical follow-up was also obtained.

Results: Thirty-one cases of DCIS or LN involving SA were obtained (12 cases of DCIS, 19 cases of LN including LCIS and ALH). Histomorphologic features commonly seen with DCIS or LN involving SA included lobulocentric architecture (31/31, 100\%), myoepithelial cells visible by $H \& E$ at least focally (31/31, 100\%), and separate areas of SA not involved by neoplasia $(29 / 31,93.5 \%)$. Features that were sometimes seen included hyaline basement membranes surrounding the lesion (14/31, $45.2 \%)$, DCIS/LN apart from the area of involvement by SA $(16 / 31,51.6 \%)$, and calcifications associated with DCIS/LN/SA (12/31, $38.7 \%)$. Features that were not commonly seen included desmoplasia $(6 / 31,19.4 \%)$, dense inflammation $(4 / 31,12.9 \%)$, and single epithelial cells enveloped by flattened myoepithelial cells $(6 / 31,19.4 \%)$. Of the ten cases of DCIS with known follow-up, four showed DCIS involving either SA or a complex SA on excision $(4 / 10,40 \%)$, four had only DCIS $(4 / 10,40 \%)$, one had DCIS with a small 1.8-mm focus of predominantly tubular carcinoma (1/10, 10\%), and one showed invasive ductal carcinoma on excision $(1 / 10,10 \%)$. The latter case of invasive ductal carcinoma occurred in a patient who had a delay of 3 years from diagnosis to surgical resection. Of the eight cases of $\mathrm{LN}$ with surgical follow-up, seven had LCIS $(7 / 8,87.5 \%)$, and one showed only fibroadenoma and SA with no 
residual LN in the excised specimen (1/8, $12.5 \%)$. Importantly, no invasive carcinoma was identified in any of the resections for LN involving SA.

Conclusions: In our series of carcinoma in situ (CIS) involving sclerosing adenosis diagnosed on core biopsy, lobular lesions involving SA were more common than ductal lesions. Ductal and lobular carcinoma in situ involving adenosis were best diagnosed by the low-power appearance of a lobulocentric pattern of growth. The most helpful diagnostic feature was the observation of additional foci of carcinoma in situ away from the adenosis. Immunohistochemical stains for myoepithelial cells were useful in particularly difficult cases. The presence of stromal desmoplasia does not preclude the diagnosis of carcinoma in situ involving adenosis. Knowledge of these diagnostic pearls can reduce over-interpretation of CIS on core biopsy and subsequent overtreatment.

Keywords: Adenosis; Breast; Core biopsy; Ductal carcinoma in situ; Lobular carcinoma in situ

\section{Key Summary Points}

Carcinoma in situ involving adenosis (both ductal carcinoma in situ and lobular carcinoma in situ) is a difficult diagnosis to make on core needle biopsy and therefore must be forefront in the mind of the diagnostician.

Awareness of the lesion and the characteristic morphology presented herein can aide in rendering the appropriate diagnosis and providing recommendations for management of this localized lesions.

When present, the recognition of additional foci of carcinoma away from the adenosis is a helpful feature.

Importantly, the presence of stromal desmoplasia does not preclude the diagnosis of carcinoma in situ involving adenosis.

\section{INTRODUCTION}

The diagnosis of ductal carcinoma in situ (DCIS) or lobular neoplasia (LN) involving a sclerosing lesion can present difficult diagnostic challenges on limited biopsy material, and this in situ disease can frequently be confused with invasive carcinoma on core biopsy. Knowledge of this diagnostic mimicker and its distinct histopathology can aid decision-making and prevent overdiagnosis and patient harm. Sclerosing adenosis (SA) alone can present difficult diagnostic challenges, especially on limited core biopsy material. The involvement of SA by ductal or LN can be particularly troublesome, since in florid cases it may mimic an invasive carcinoma. However, if the possibility of in situ neoplasia involving SA is considered, the diagnosis can be straightforward. Certainly immunohistochemistry can aid in the differential diagnosis, but if adenosis is not initially considered by the practicing pathologist, the opportunity to even employ immunohistochemistry to arrive at the correct diagnosis will be lost.

The purpose of this study was to provide awareness of the differential diagnosis of CIS involving adenosis and decrease the incidence of over-interpretation of this lesion on core biopsy and subsequent overtreatment. Herein we provide histological criteria to define features of ductal and lobular neoplasia involving sclerosing adenosis on core biopsy, and examine the clinicopathological features of this disease occurring in patients. To the best of our knowledge, this series represents the largest to date of such lesions identified on core biopsy with follow-up and serves to highlight this difficult diagnosis and diagnostic pearls necessary to avoid overtreatment.

\section{METHODS}

Our computer files were searched for patients who had undergone core needle biopsies of mammographic abnormalities, specifically cases of breast core needle biopsy specimens with a dominant diagnosis of sclerosing adenosis involved by either DCIS or LCIS/atypical lobular 
hyperplasia (ALH) from 1999 to present. Cases with concomitant invasive carcinoma were excluded. We obtained 31 cases: 12 cases of DCIS and 19 cases of LN (for the purpose of this paper, no distinction was made between LCIS and ALH). The slides were reviewed by two pathologists, both with expertise in breast neoplasia (DR, LM). Information about clinical presentation and follow-up and/or resection was also obtained. This was a lab-based and quality improvement project, and as such did not meet institutional review board criteria for review.

\section{RESULTS}

The average patient age was 54.1 years, with an age range of 21-65 years. Presenting clinical findings included masses $(6 / 31,19.4 \%)$, calcifications on mammography (21/31, 67.7\%), architectural asymmetry or distortion on mammography $(3 / 31,9.7 \%)$, and an area of enhancement on MRI (1/31, 3.2\%).

The lesions were sampled by ultrasoundguided core biopsy $(6 / 31,19.4 \%)$, stereotacticguided core biopsy $(21 / 31,67.7 \%)$, MRI-guided core biopsy $(2 / 31,6.5 \%)$, or by biopsy not specified $(2 / 31,6.5 \%)$. For stereotactic-guided core biopsy, the cores ranged from 9- to 11-gauge, and the number of cores varied from 5 to 18 . For ultrasound-guided core biopsy, the cores ranged from 16- to 18-gauge, and the number of cores ranged from 4 to 5 . The two MRI-guided biopsies were both 9-gauge, and 12 cores were obtained in each procedure.

Of a total of 31 cases of SA, 12 showed DCIS and $19 \mathrm{LN}$. Of the 12 patients with DCIS involving SA diagnosed on core needle biopsy, four underwent total mastectomy, six had segmental mastectomy, and two patients were lost to follow-up. Ten of the 12 cases with known follow-up reported that four had DCIS involving either SA or a complex sclerosing lesion (4/ $10,40 \%)$, four had only DCIS $(4 / 10,40 \%)$, one had DCIS with a small $1.8-\mathrm{mm}$ focus of predominantly tubular carcinoma $(1 / 10,10 \%)$, and one showed invasive ductal carcinoma on excision $(1 / 10,10 \%)$, which occurred in a patient who had a delay of 3 years from diagnosis to surgical resection (Table 1).

Patients with high-risk lesions, including lobular neoplasia involving SA, were presented at our multi-team clinical management conference that includes surgery, cancer prevention, pathology, and radiology. Patients with DCIS involving SA were assigned to surgical excision without discussion in the high-risk conference. For each patient, either chemoprevention, excision, or radiologic follow-up at regular intervals was recommended, based on clinical factors such as whether persistent calcification remained or whether a radiologic/pathologic discordance was observed. Patients with a positive family history for breast cancer (5) were also counseled to consider excision. Following the clinical management conference, four patients underwent total mastectomy $(4 / 19,21.1 \%)$, four patients had excisional/segmental biopsies $(4 / 19,21.1 \%)$, nine had radiologic follow-up only $(9 / 19,52.6 \%)$, and one has not had final disposition $(1 / 19,5.3 \%)$ (recent case). Of the patients with surgical follow-up (8 patients), seven had LCIS $(7 / 8,87.5 \%)$ and one showed only fibroadenoma and SA with no lobular neoplasia in the specimen $(1 / 8,12.5 \%)$. No invasive carcinoma was identified in these latter patients undergoing surgical excision.

\section{PATHOLOGY}

Histomorphologic features commonly seen with DCIS/LN involving SA included lobulocentric architecture as a pattern of growth (31/ $31,100 \%)$, thin, attenuated myoepithelial cells visible by hematoxylin and eosin (H\&E) stain at least focally when focusing up and down on the lesion $(31 / 31,100 \%)$, and adjacent areas of SA not involved by neoplasia (29/31, 93.5\%) (Figs. 1, 2). Features that were sometimes seen included hyaline basement membranes surrounding the lesion $(14 / 31,45.2 \%)$, ductal or lobular neoplasia apart from the area of involvement by SA $(16 / 31,51.6 \%)$, and calcifications associated with DCIS/LN/SA (12/31, $38.7 \%)$. Features that were observed but not commonly seen included desmoplasia (6/31, $19.4 \%)$, dense inflammation $(4 / 31,12.9 \%)$, and 
Table 1 Clinical characteristics and follow-up of 31 cases of sclerosing adenosis involved by carcinoma in situ

\begin{tabular}{|c|c|c|c|c|c|c|}
\hline Case & $\begin{array}{l}\text { Age } \\
\text { (years) }\end{array}$ & $\begin{array}{l}\mathbf{D} / \\
\mathbf{L}\end{array}$ & Presentation & Treatment & Findings & Follow-up \\
\hline 1 & 61 & $\mathrm{~L}$ & $\mathrm{C}$ & SM & SA/LCIS & A\&W 9 years \\
\hline 2 & 61 & $\mathrm{~L}$ & $\mathrm{C}$ & SM & SA/LCIS & A\&W 9 years \\
\hline 3 & 56 & $\mathrm{~L}$ & $\mathrm{C}$ & NS & $\mathrm{N} / \mathrm{A}$ & A\&W 5 years \\
\hline 4 & 54 & $\mathrm{~L}$ & M & SM & FA, SA only & A\&W 6 years \\
\hline 5 & 53 & $\mathrm{~L}$ & $\mathrm{C}$ & $\mathrm{TM}$ & SA/LCIS, ADH & No $F / U$ \\
\hline 6 & 36 & $\mathrm{~L}$ & $\mathrm{C}, \mathrm{A}$ & NS, T & $\mathrm{N} / \mathrm{A}$ & A\&W 6 years \\
\hline 7 & 57 & $\mathrm{~L}$ & $\mathrm{C}$ & NS & $\mathrm{N} / \mathrm{A}$ & No F/U \\
\hline 8 & 55 & $\mathrm{~L}$ & $\mathrm{C}$ & NS, T & $\mathrm{N} / \mathrm{A}$ & A\&W 5 years \\
\hline 9 & 55 & $\mathrm{~L}$ & $\mathrm{C}$ & NS & $\mathrm{N} / \mathrm{A}$ & NSCLC, no breast $\mathrm{F} / \mathrm{U}$ \\
\hline 10 & 54 & $\mathrm{~L}$ & $\mathrm{C}$ & NS, T & $\mathrm{N} / \mathrm{A}$ & A\&W 6 years \\
\hline 11 & 60 & $\mathrm{~L}$ & $\mathrm{C}$ & NS & $\mathrm{N} / \mathrm{A}$ & $\mathrm{A} \& W 2$ years \\
\hline 12 & 43 & $\mathrm{~L}$ & $\mathrm{C}$ & NS & $\mathrm{N} / \mathrm{A}$ & $\mathrm{A} \& \mathrm{~W} 1$ year \\
\hline 13 & 47 & $\mathrm{~L}$ & E & $\mathrm{TM}$ & SA/LCIS & $\mathrm{A} \& W 2$ years \\
\hline 14 & 48 & $\mathrm{~L}$ & $\mathrm{C}$ & $\mathrm{TM}$ & LCIS & $\mathrm{A} \& \mathrm{~W} 1$ year \\
\hline 15 & 57 & $\mathrm{~L}$ & $\mathrm{D}$ & SM & SA/LCIS & $\mathrm{A} \& \mathrm{~W} 1$ year \\
\hline 16 & 21 & $\mathrm{~L}$ & M & NS & $\mathrm{N} / \mathrm{A}$ & US F/U \\
\hline 17 & 45 & $\mathrm{~L}$ & $\mathrm{C}$ & $\mathrm{TM}$ & LCIS & $\mathrm{A} \& \mathrm{~W}<1$ year \\
\hline 18 & 51 & $\mathrm{~L}$ & $\mathrm{C}$ & Pending & N/A & N/A \\
\hline 19 & 65 & $\mathrm{~L}$ & $\mathrm{C}$ & NS & $\mathrm{N} / \mathrm{A}$ & $\mathrm{A} \& \mathrm{~W} 6 \mathrm{mo}$. \\
\hline 20 & 49 & $\mathrm{D}$ & $\mathrm{D}$ & $\mathrm{TM}$ & $\begin{array}{l}\text { DCIS, LCIS, } 1.8-\mathrm{mm} \text { focus of inv. Ca } \\
\text { (pred. tubular type) }\end{array}$ & $\mathrm{A} \& \mathrm{~W} 11$ years \\
\hline 21 & 66 & $\mathrm{D}$ & $\mathrm{C}, \mathrm{Di}$ & SM & DCIS/CSL & A\&W 4 years \\
\hline 22 & 49 & $\mathrm{D}$ & $\mathrm{C}$ & $\mathrm{TM}$ & IDC, $3 \mathrm{~cm}$ with SA/DCIS & No $F / U$ \\
\hline 23 & 34 & $\mathrm{D}$ & $\mathrm{C}$ & TM & DCIS & No F/U \\
\hline 24 & 72 & $\mathrm{D}$ & $\mathrm{C}$ & $\mathrm{TM}$ & DCIS & Deceased, small cell ca \\
\hline 25 & 55 & $\mathrm{D}$ & M & SM & SA/DCIS & $\mathrm{A} \& W \mathrm{~W} 4$ years \\
\hline 26 & 52 & $\mathrm{D}$ & A & SM & SA/DCIS & A\&W 2 years \\
\hline 27 & 46 & $\mathrm{D}$ & $\mathrm{C}$ & UNK & UNK & UNK \\
\hline 28 & 64 & $\mathrm{D}$ & $\mathrm{C} / \mathrm{D}$ & SM & DCIS & $\mathrm{A} \& \mathrm{~W} 1$ year \\
\hline 29 & 58 & $\mathrm{D}$ & M & UNK & UNK & UNK \\
\hline 30 & 47 & $\mathrm{D}$ & M & SM & DCIS & $\begin{array}{l}\text { Also IDC another site, } \\
\text { same breast }\end{array}$ \\
\hline
\end{tabular}


Table 1 continued

\begin{tabular}{lllllll}
\hline Case & $\begin{array}{l}\text { Age } \\
\text { (years) }\end{array}$ & $\begin{array}{l}\text { D/ } \\
\text { L }\end{array}$ & Presentation & Treatment & Findings & Follow-up \\
\hline 31 & 55 & D & M & SM & DCIS/CSL & A\&W 2 years \\
\hline
\end{tabular}

$A$ asymmetry, $D$ ductal, $L$ lobular, $D i$ distortion, $S M$ segmental mastectomy, $T M$ total mastectomy, $N S$ no surgery, $T$ tamoxifen, $I D C$ invasive ductal carcinoma, $U N K$ unknown, $A \& W$ alive and well, $C S L$ complex sclerosing lesion, $N S C L C$ non-small cell lung carcinoma

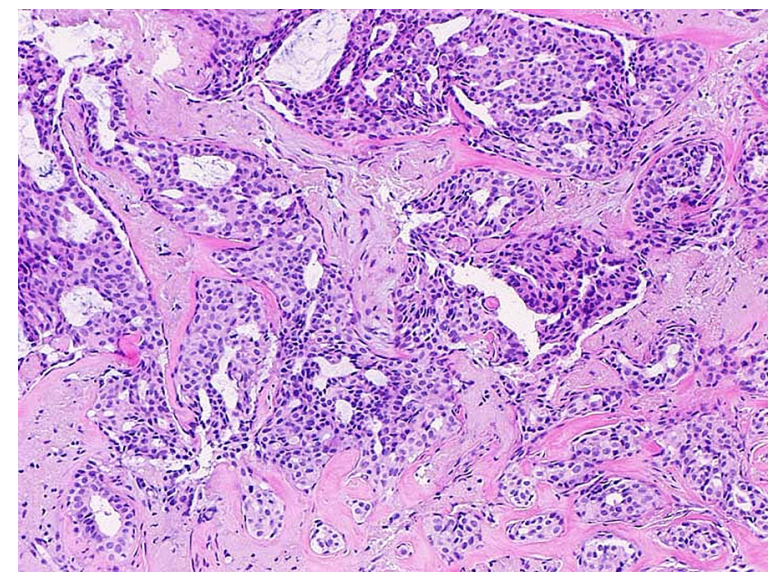

Fig. 1 Ductal carcinoma in situ (DCIS) involving sclerosing adenosis, H\&E $\times 100$. Note pink hyalinized stroma and flattened myoepithelial cell layer along the periphery of the in situ carcinoma

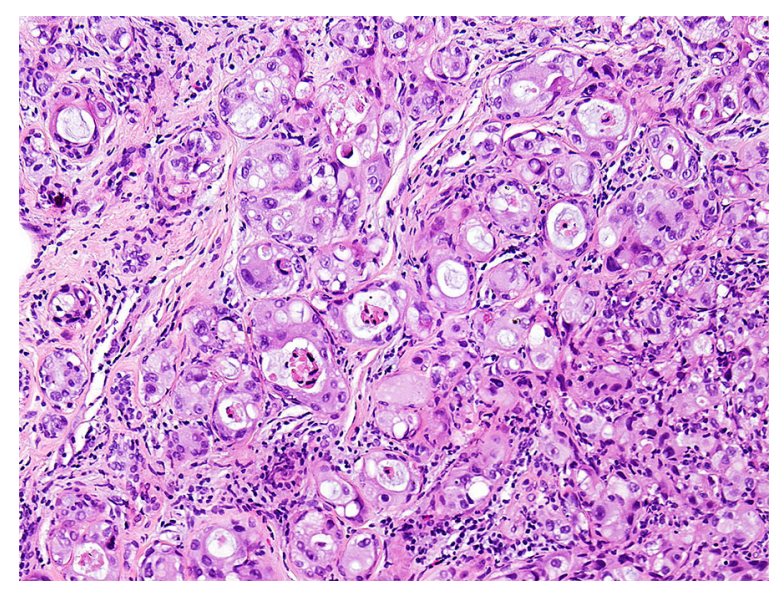

Fig. 2 Ductal carcinoma in situ (DCIS) involving sclerosing adenosis, H\&E $\times 200$. Note inconspicuous flattened myoepithelial encasing the high grade carcinoma in situ cell layer and peri-lobular inflammation

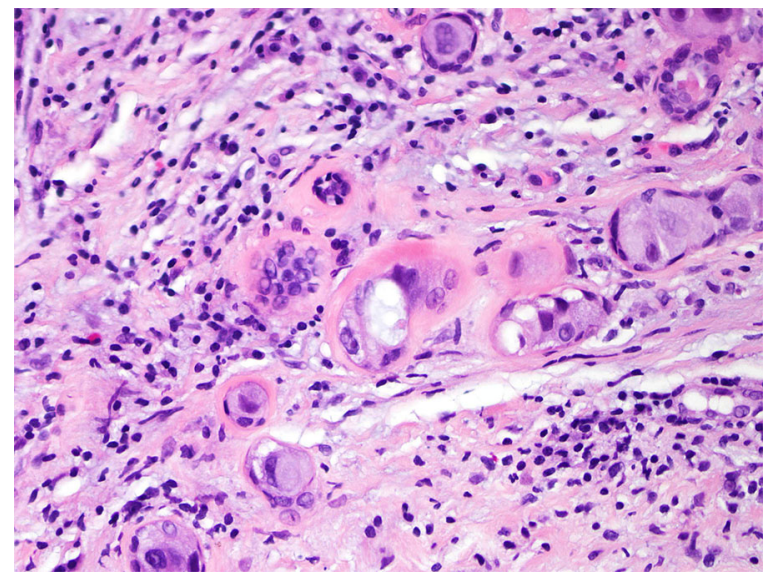

Fig. 3 High-power magnification of DCIS involving sclerosing adenosis with myoepithelial cells enveloping single neoplastic cells and cell clusters. $\mathrm{H} \& \mathrm{E} \times 400$

Table 2 Histologic characteristics of carcinoma in situ involving sclerosing adenosis

\begin{tabular}{ll}
\hline Feature & \% of cases \\
\hline Lobulocentric pattern & 100 \\
ME cells visible & 100 \\
Separate areas of SA & 93.5 \\
Separate areas of DCIS/LN & 51.6 \\
Hyaline basement membranes & 45.2 \\
Calcifications & 38.7 \\
Desmoplasia & 19.4 \\
Single cells enveloped by ME cells & 19.4 \\
Inflammation & 12.9 \\
\hline
\end{tabular}

$M E$ myoepithelial, $S A$ sclerosing adenosis, DCIS ductal carcinoma in situ, $L N$ lobular neoplasia 


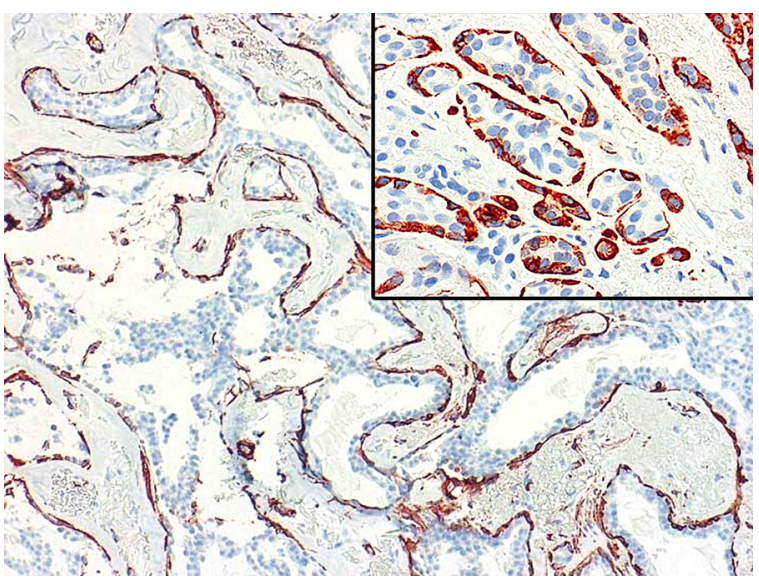

Fig. 4 Smooth muscle actin immunohistochemistry stain highlights myoepithelial cells enveloping the DCIS

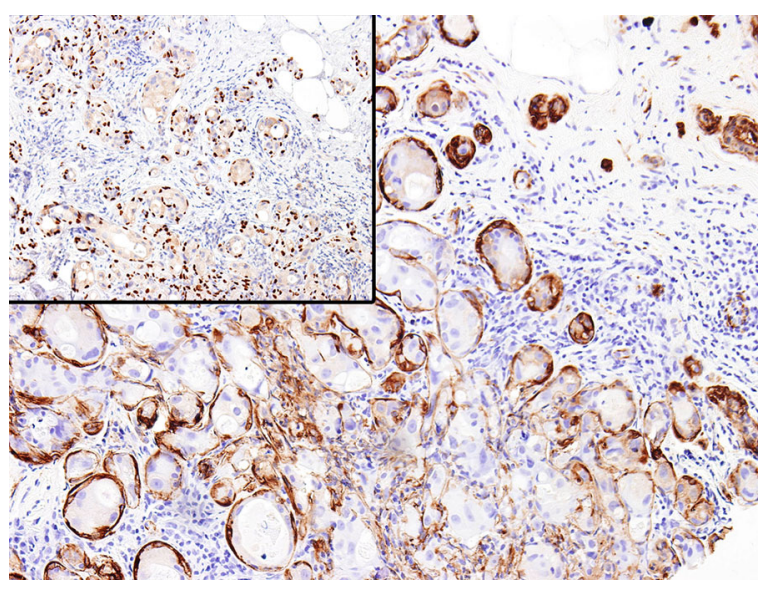

Fig. 5 Smooth muscle actin immunohistochemistry stain highlights myoepithelial cells enveloping the DCIS involving adenosis. Inset: p63 immunohistochemical stain shows nuclear immunoreactivity of myoepithelial cells surrounding DCIS

single cells enveloped by myoepithelial cells (6/ 31, 19.4\%) (Fig. 3) (Table 2).

Immunohistochemistry was used in occasional cases to exclude invasive carcinoma (9/ $31,29.0 \%$ ), and included a combination of smooth muscle actin (Sigma, 1:80,000 dilution), smooth muscle myosin heavy chain (Invitrogen, 1:200 dilution) and/or P63 (Biocare, dilution 1:1000) (Figs. 4, 5). Appropriate quality checks and controls were utilized according to manufacturer standards. E-cadherin (Life Technologies, 1:1000 dilution) was employed in $6 / 31$ cases $(19.4 \%)$ to differentiate between

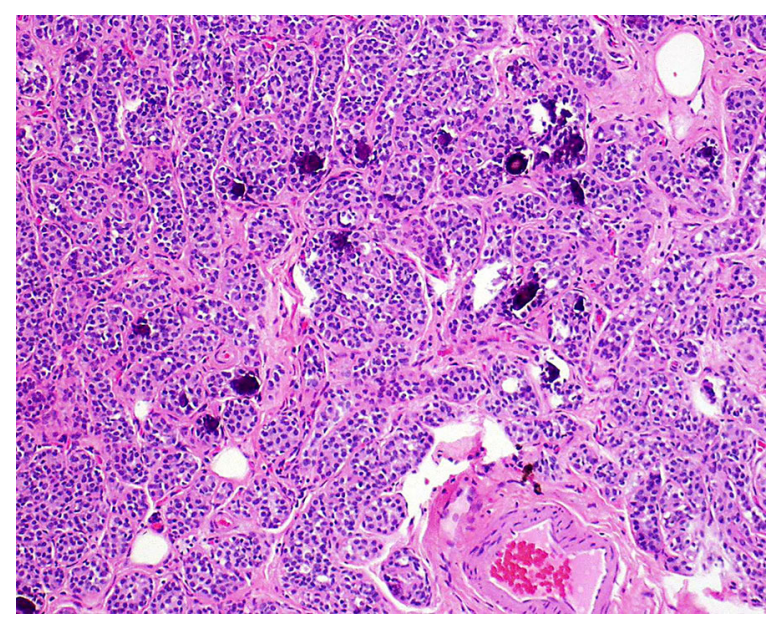

Fig. 6 Lobular neoplasia involving sclerosing adenosis. Notice nesting pattern of non-cohesive bland uniform cells distending the sclerosing adenosis and minimizing the hyalinized pink stroma. Coarse magenta microcalcifications are easily identified and were likely the impetus for biopsy

ductal and lobular neoplasia (Fig. 6) involving sclerosing adenosis. Cases of DCIS exhibited strong membranous E-cadherin staining of the cells comprising the intraductal carcinoma, while E-cadherin staining was absent in the neoplastic cells of LCIS.

\section{DISCUSSION}

Core needle biopsy interpretation is a reliable method for triage of mammographic abnormalities. However, the diagnosis of DCIS or lobular neoplasia involving a sclerosing lesion on core biopsy may present difficult diagnostic challenges and frequently can be confused with invasive carcinoma, leading to over-management including inappropriate administration of chemotherapy or more extensive surgery. However, when one is familiar with this diagnostic pitfall, the diagnosis becomes much more straightforward, and immunohistochemical stains are not necessary for confirmation of the diagnosis in most cases.

A review of the English literature revealed four single case reports and four case series reports, overall describing 39 total cases of DCIS and/or LN involving SA. Our case series adds 31 
additional cases to the literature. In the first described series of six cases of LCIS involving SA [1], Fechner et al. found that the architecture of the lesion by low-power assessment was the key to the correct diagnosis, noting that an invasive carcinoma should have no overall organizational pattern. We also found low-power assessment to be the most useful clue that one is dealing with an SA. Sclerosing lesions maintain an organized, lobular architectural pattern. If one is concerned about invasive carcinoma but the lesion maintains a lobular architecture at low power, the possibility of DCIS/LN involving an SA should be strongly considered.

In a later case series which included both ductal and lobular cases [2], Rasbridge et al. commented that the lobular architecture was sometimes difficult to fully assess due to distortion by both SA and ductal or lobular neoplasia, and that both immunohistochemical stains and comparison with adjacent adenosis not involved by ductal or lobular neoplasia were helpful. We also found that the lobular architecture is sometimes difficult to assess on core biopsy, particularly if the lesion of concern is at the edges of the core biopsy. One extremely helpful clue in the diagnosis of these cases is the presence of SA not involved by neoplasia in other portions of the biopsy. This feature was found in almost all cases, and provided a reassuring "internal control" of the process for comparison. This finding also provides proof that a sclerosing process is occurring within the breast. Another helpful clue occurs when the neoplastic process does not entirely fill the sclerosing lesion. When this occurs the periphery of the lobule commonly shows the recognizable histology of a usual sclerosing lesion. In our experience, if this finding is seen, invasion is very unlikely.

In difficult cases, immunohistochemical staining for myoepithelial markers may be employed. For instance, some of our cases displayed occasional neoplastic single cells enveloped by myoepithelial cells (identified within the center of the lobule). Confirmatory myoepithelial markers should be ordered in these cases to exclude invasive carcinoma. Another instance in which immunohistochemical stains should be considered is when the sclerosing lesion is associated with a dense inflammatory background. In these cases, both myoepithelial markers and a pan-cytokeratin stain may be helpful to confirm the diagnosis of in situ carcinoma.

In a smaller mixed case series [3], Eusebi et al. noted that the presence of a PAS-positive basal lamina and myoepithelial cells were useful adjuncts to the low-power assessment. We also noted that the presence of myoepithelial cells in at least some portions of the lesion was a helpful feature. Often the myoepithelial cells are thin or attenuated and are not seen at first glance. Focusing up and down on the tissue plane (or immunohistochemical stains) may be necessary.

Of course, the reason that these lesions are so important to consider is the real possibility of confusion with an invasive carcinoma. In the mixed case series [3] by Eusebi et al., two of the patients went to mastectomy because they were originally diagnosed as invasive carcinoma by frozen section. Other individual case reports [4-7] were notable for a case of LCIS/SA that was originally called invasive carcinoma on biopsy. Some of our cases demonstrated characteristics which might normally be considered to be associated with an invasive process, if the overall architectural clues are not recognized. Desmoplasia was seen in $19.4 \%$ of cases, which to our knowledge has never been described in association with these lesions. In addition, single cells enveloped by thin, attenuated myoepithelial cells also were present within the center of the sclerosing lesion in $19.4 \%$ of cases. In all cases, attention to the other criteria helped to prevent misdiagnosis based on a single characteristic.

Follow-up data were given for some of the previous case studies. In the six cases of LCIS involving SA [1], Fechner et al. found that the only invasive carcinoma was an incidental tubular carcinoma, which was located away from areas of SA. In a later case series which included both ductal and lobular cases [2], Rasbridge et al. did not provide patient followup, but noted that three cases had small foci of invasive carcinoma in addition to ductal or lobular neoplasia involving SA. In the mixed case series [3] by Eusebi et al., the patients were 
all alive and well after quadrantectomy or mastectomy, for a follow-up time ranging from 23 months to 10 years. In a small mixed case series [8], Oberman et al. selected cases of DCIS/ LCIS which appeared solely in sclerosing adenosis, with no carcinoma in situ present outside the sclerosing lesion. Eight of the patients were followed for an average of 3 years, and were alive and well. As expected, our follow-up data also indicate that these patients do extremely well. Of the patients with invasive ductal carcinoma on excision, one patient waited 3 years between diagnosis and excision, with only alternative therapies in the interim. The only unexpected case of invasive carcinoma on surgical follow-up was a $1.8-\mathrm{mm}$ focus of carcinoma which was predominantly of tubular type, and the patient has been alive and well for 11 years since the diagnosis. Of the patients with LCIS, all are alive and well (with the exception of two patients who had a history of primary lung carcinoma). Despite the known increased incidence of invasive carcinoma in these patients, none of the patients with LCIS involving SA who underwent surgery had invasive carcinoma in their excisions.

The previously reported cases included 21 lobular, 15 ductal, and 3 combined ductal/lobular neoplasia cases. Our cases showed a very slight lobular predominance (19 lobular, 12 ductal), a relationship also described by Koerner [9]. One might expect that because sclerosing adenosis is an estrogen-dependent lobulocentric process, the incidence of associated lobular neoplasia would be much higher, but this has not been demonstrated. One must be careful not to assume that a low-grade carcinoma in situ involving sclerosing adenosis is lobular carcinoma, because ductal cancerization is almost as frequent. Evaluating for secondary lumen formation (even in adenosis) and cell polarization is helpful. An E-cadherin stain can be employed to help discriminate between the two entities.

The limitations of this study include the small sample size of patients who have this uncommon but clinically relevant diagnosis. Another limitation of the study is the lack of extensive follow-up, although among patients with DCIS who underwent excision, 90\% had either DCIS, or DCIS with microinvasion. The limited number of cases only allow for descriptive comparisons of the histology. Using descriptive statistics, the top three features (lobulocentric pattern, visibility of myoepithelial cells, and separate areas of sclerosing adenosis) are statistically more frequently occurring than what is randomly expected. Core needle biopsy interpretation of mammographic abnormalities is a reliable method of assessment, and diagnosticians must maintain a broad differential diagnosis that includes mimickers of invasive carcinoma to prevent over-diagnosis and overtreatment.

\section{CONCLUSION}

In summary, we present the largest series of 31 cases of SA involved by DCIS or lobular neoplasia initially diagnosed on core biopsy. In addition to the low-power architectural pattern that may be useful in making the diagnosis, the pathologist should focus on the attenuated peripherally located myoepithelial cells and search for carcinoma in situ in other areas of the biopsy material. Importantly, desmoplasia may be identified in cases of sclerosing adenosis, and its presence does not indicate invasive carcinoma, as in our series only $19.4 \%$ of cases demonstrated this finding. Knowledge of these diagnostic pearls will hopefully prevent erroneous diagnosis and overmanagement of patients with in situ disease.

\section{ACKNOWLEDGEMENTS}

We thank the patients who entrust us with their care and whose specimens were analyzed for this study.

Funding. No funding or sponsorship was received for this study or publication of this article.

Authorship. All named authors meet the International Committee of Medical Journal Editors (ICMJE) criteria for authorship for this article, take responsibility for the integrity of the work as a whole, and have given their approval for this version to be published. 
Prior presentation. This abstract was submitted in part at the United States and Canadian Academy of Pathology annual meeting in 2011.

Disclosures. Dana Richards, Alberto A. Ayala, and Yun Wu declare that they have no conflict of interest. Lavinia Middleton is on the editorial board of this journal but has nothing further to disclose.

Compliance with Ethics. This study was excluded by our institutional review board for human subject research, as it is a lab-based protocol study that reviewed outcomes of mastectomy and segmental biopsy diagnoses compared with core needle biopsy results (Quality Assurance).

Open Access. This article is distributed under the terms of the Creative Commons Attribution-NonCommercial 4.0 International License (http://creativecommons.org/licenses/ by-nc/4.0/), which permits any noncommercial use, distribution, and reproduction in any medium, provided you give appropriate credit to the original author(s) and the source, provide a link to the Creative Commons license, and indicate if changes were made.

Open Access. This article is licensed under a Creative Commons Attribution-NonCommercial 4.0 International License, which permits any non-commercial use, sharing, adaptation, distribution and reproduction in any medium or format, as long as you give appropriate credit to the original author(s) and the source, provide a link to the Creative Commons licence, and indicate if changes were made. The images or other third party material in this article are included in the article's Creative Commons licence, unless indicated otherwise in a credit line to the material. If material is not included in the article's Creative Commons licence and your intended use is not permitted by statutory regulation or exceeds the permitted use, you will need to obtain permission directly from the copyright holder. To view a copy of this licence, visit http://creativecommons.org/licenses/by$\mathrm{nc} / 4.0 /$.

\section{REFERENCES}

1. Fechner RE. Lobular carcinoma in situ in sclerosing adenosis. A potential source of confusion with invasive carcinoma. Am J Surg Pathol. 1981;5(3):233-9.

2. Rasbridge SA, Millis RR. Carcinoma in situ involving sclerosing adenosis: a mimic of invasive breast carcinoma. Histopathology. 1995;27(3):269-73.

3. Eusebi V, Collina G, Bussolati G. Carcinoma in situ in sclerosing adenosis of the breast: an immunocytochemical study. Semin Diagn Pathol. 1989;6(2): 146-52.

4. Ichihara S, Aoyama H. Intraductal carcinoma of the breast arising in sclerosing adenosis. Pathol Int. 1994;44(9):722-6.

5. Chan JK, Ng WF. Sclerosing adenosis cancerized by intraductal carcinoma. Pathology. 1987;19(4):425-8.

6. Visscher DW. Apocrine ductal carcinoma in situ involving a sclerosing lesion with adenosis: report of a case. Arch Pathol Lab Med. 2009;133(11):1817-21.

7. Jung WH, Noh TW, Kim HJ, Kim DY, Lee HD, Oh KK. Lobular carcinoma in situ in sclerosing adenosis. Yonsei Med J. 2000;41(2):293-7.

8. Oberman HA, Markey BA. Noninvasive carcinoma of the breast presenting in adenosis. Mod Pathol. 1991;4(1):31-5.

9. Koerner F, Maluf H. Uncommon morphologic patterns of lobular neoplasia. Ann Diagn Pathol. 1999;3(4):249-59. 